\title{
Research Regarding Analysis of the Target Safety Standard for Fire Resistance Design and its Mode of Expression -Estimation for the Safety Standard Targeted by the Verification Method for Fire Resistance Performance-
}

\author{
Akiko Natori \\ Non-Life Insurance Rating Organization of Japan, Japan
}

\section{Chapter 1 INTRODUCTION}

\subsection{Introduction of Verification Method for Fire Resistance Performance and its Technical Issues}

\subsubsection{Transition of the Building Standards Law (Japan)}

The Building Standards Law of Japan (BSL, hereafter) was enacted in 1950, and has been improved in response to advancements in construction engineering and changes in society. In the 1960s, when large-scale buildings were actively being built everywhere, many fires in such buildings took place and resulted in tremendous number of victims due to smoke spread to upper floors from the floor of fire origin through vertical shafts such as elevators, staircases and ducts. In response to this situation, a regulation for shaft compartment was added to the enforcement order in 1969. In the following year (1970), in response to the increasing construction of high-rise and large-scale buildings, more regulations were added with respect to smoke control systems, emergency lighting systems, rescue entrances and emergency elevators. With this background, the building standards law was extended for fire disaster prevention and evacuation safety. However, in the early 1970s, soon after the regulation enhancement, catastrophic fire disasters successively occurred in 1972 (Sen'nichi Department Store Fire, 118 fatalities) and in 1973 (Taiyou Department Store Fire, 103 fatalities). The reason for this is because the new regulations were not applied to existing buildings constructed prior to regulation enhancement. These fire accidents revealed deficiencies in the regulations relating to fires in buildings. Later, as the lessons from the past fire accidents were learned, the fire prevention regulations was revised and improved further.

During the development and extension of regulations, fire safety measures were described by prescriptive way without explaining the reasons why such safety measures are needed to achieve a certain level of safety. Specific provisions were prescribed and mandated as if they were the only possible solution. In addition, the provisions were devised based on past experiences rather than scientific and engineering evidence. As for the principal construction members of fire-resistive buildings, required fire resistance 
time was prescribed depending on the position of construction elements and number of supporting floors counted from top (Enforcement order, Article 107). Building designers were allowed only to select method of construction among approved specifications. In the old days when the construction materials and methods were limited, prescriptive code was a simple and rational way to archive fire safety. Today, it is difficult and irrational to apply prescriptive solutions to large and complex buildings constructed with innovative materials and construction methods.

In order to improve the situation and to improving the fire safety design based on engineering discipline, the Ministry of Construction (former name of MLIT, Ministry of Land, Infrastructure, Transport and Tourism) initiated a comprehensive research project entitled "Development of Total Fire Prevention Design Methods for Building (1982-1986)" which is known as Bouka-Sopro). The achievements of the project was not directly reflected in revision of the BSL, but proposed engineering design system was accepted by way of equivalency clause of BSL (Article 38), which defines the approval process of the Minister of Construction. The engineering methods significantly raised the awareness of the construction industry, and spread in practice with the help of the publication of guideline document[1].

As the engineering method get certain publicity, BSL was revised in the year of 2000 for further adoption of performance concept. While the equivalency term was abolished, performance requirements and associated verification methods were newly introduced into the BSL and corresponding notifications[2].

\subsubsection{Concept of the Performance-based System and Verification Methods for Fire Safety}

Figure 1.1.1 shows the comparison between the prescriptive and performance-based systems[3]. This 5-layer system illustrates the basic concepts needed to comprehend the performance-based regulation systems. The left side corresponds with prescriptive system up to 2000 and the right side describes the performance-based system.

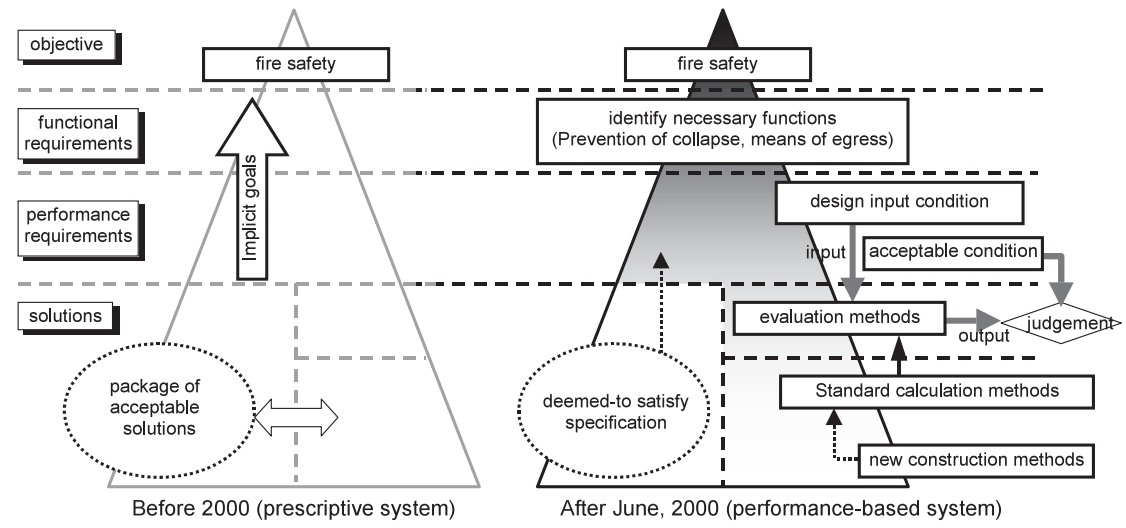

Figure 1.1.1 Comparison of the prescriptive and performance-based system in NKB format 
The measures for achieving the required fire safety levels are packaged in the prescriptive system. The package implicitly specifies the fire safety; however, the intention and required level is not clarified. Therefore, the target of the design is to fit it into the specification. In contrast, in the performance-based system, the intention of fire safety is specified first. Secondly, in order to meet the target specifications, the functional requirement are listed, and the required level of the performance-based criterion per function is described. The goal of fire safety design is to meet the performance requirement, and the means to comply with the requirements can be arbitrarily chosen. On the other hand, the package for the prescriptive system remains unaltered.

Therefore, Article 38 was deleted when the BSL was revised in 2000, and three methods of compliance are identified for fire prevention requirements in BSL: Route A with the prescribed standard, Route B with performance-based concept following to simple verification method and Route $\mathrm{C}$ with full utilization of performance concept followed by approval of the Minister. Two verification methods are attached to Route B, one of which is a calculation for verifying the fire resistance (Performance Verification for Fire Resistance), and the other one is a calculation for verifying the evacuation safety (Verification for Evacuation Safety). Each verification method is used for proving the efficiency of the fire safety measures. Both verification methods comprise many simplified formulas, and the combination of fire safety provisions can be verified quantitatively[4].

\subsubsection{The Concept of a Verification Method for Fire Resistance Performance and Associated Problems}

The Verification Method for Fire Resistance Performance (hereinafter, the Verification Method) is used for verifying whether principal construction elements will retain their fire resistance if the building undergoes an anticipated fire[5]. Here, the principal structural parts, comprising columns and beams, are required to have sufficient structural stability; based on the notion that none of the members should collapse. In other words, all members of the principal structural elements need to pass the verification, and the point where one of them collapses is considered as the safety limit of the entire building. In the Verification Method, the fire resistance performance of the structural members is verified by putting the deterministic input conditions into the specified formula. It should be checked whether the final result deterministically meets the requirements set in the Verification Method. In this verification framework, it is not certain which level of safety is achieved even if the requirements are satisfied. As the characteristic input values are deterministically provided, the values vary within a certain range in reality. Thus it is supposed that that some of the input values and formulas include implicit safety factors. The input value and the formula for each input parameter are specified based on the results of past research and surveys. For example, characteristic fire behavior is set severer than the average. The characteristic structural strength is set to smaller values than the actual strength. However, the concept of safety 
margins are not necessarily the same in fire engineering and structural engineering, and the safety factors with respect to external forces, the bearing capacity and the magnitude of the influence to the limit state are unknown.

For rational engineering-based designs, a necessary safety level should be set as the design target. Thus the safety factors should be appropriately selected considering the magnitude and uncertainty of each input parameter. However, in the current verification method, no such design methodology is adapted, and the archived safety level cannot be recognizable by the designers and the owners of the building. Even among specialists, such as fire engineers and structural engineers, the whole picture is hardly understood.

\subsection{Quantitative Representation of Safety Level}

\subsubsection{Basic Theory of Structural Reliability Engineering [6]}

In the field of building structures, the theory of structural reliability is commonly used. Structural reliability engineering is a branch of the reliability engineering field whose concept is to present the reliability (see Note 1) of a structure in a quantitative manner based on the probability theory. "Structure" in structural reliability engineering refers to a machine, equipment, device or structural object. Structural reliability indicates the reliability of their capacity in terms of strength and resistance. Structural reliability engineering is widely employed not only in the field of buildings, but also in machinery, material science, and nuclear and civil engineering.

\section{(1) Probability of Failure and Safety Index}

The structural reliability is quantified by the probability that the structure does not fail (or become damaged). However, probability of failure $P_{f}$, is commonly used instead of probability of successful condition. In order to obtain the probability of failure, it is necessary to define and formulate the limit state of the structure. In general, limit state function $G$ is expressed as follows

$$
G\left(x_{1}, x_{2}, \ldots, x_{n}\right) \leq 0
$$

where $x_{1}, x_{2}, \ldots x_{n}$ are input parameters regarding the limit state. If $G>0$, then it indicates a safe state, if $G<0$, then it indicates an unsafe state. If $G=0$, it indicates the limit state. Given the limit state function, the probability of failure $P_{f}$ is expressed as follows

$$
P_{f}=P_{r}[G<0]
$$

Here, the function $\operatorname{Pr}[$ func.] expresses the probability with which the event func. occurs.

The probability of failure can be obtained if the probability distribution functions (PDF) for input parameters are given. However, the probability of failure is very small compared to 1 . In order to calculate the probability of failure accurately, it is necessary Note 1 According to the JIS[7] definition, this is defined as "a property that can fulfill a required function under given conditions of an item for a specified period of time". 
to know the tails of PDF for all the input parameters. As the tails of parameters are difficult to determine in most of the systems, a safety index is used as an alternative measure to the probability of failure. The safety index is a measure of distance from average to limit state normalized by standard deviation. For example, if a limit state function is estimated as shown in Figure 1.2.1, the safety index is calculated by average value $\left(\mu_{G}\right)$ and the standard deviation $\left(\sigma_{G}\right)$ of $G$ as

$$
\beta=\frac{\mu_{\mathrm{G}}}{\sigma_{\mathrm{G}}}
$$

In Figure 1.2.1, the portion under the curve shaded in black is an unsafe area. This area corresponds to the probability of failure $P_{f}$. When the limit state function $G$ follows normal distribution, the probability of failure and the safety index are correlated as

$$
P_{f}=1-\Phi(\beta)
$$

Here, $\Phi$ is a standard normal distribution of the density function (normal distribution: average value 0 and standard deviation.

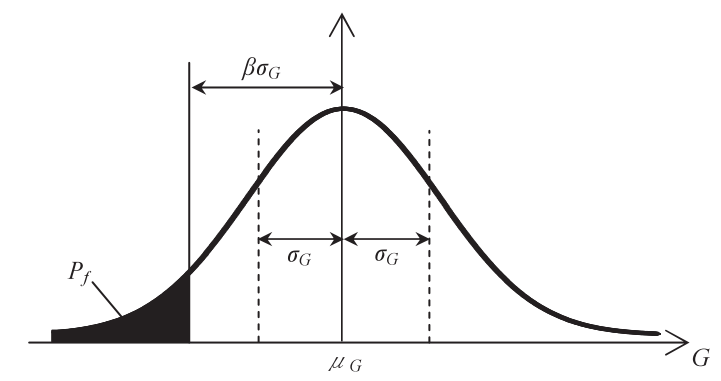

Figure 1.2.1 The probability density function of $G$ and the safety index

(2) Design Point and Partial Safety Factor of Input Parameter [8-13]

In this section, a method for obtaining the probability of failure and the safety index is formulated based on the method of structural reliability engineering. By taking an example from the relation between strength and working load, the limit state function is defined as follows

$$
G=R-S
$$

where $R$ is a resistance parameter (member strength) and $S$ is a load parameter (load on that member (corresponds to the force of a fixed load, movable load, earthquake load and wind load)).

$G=0$ indicates a limit state where the member strength and the working load are balanced. $G>0$ indicates a safe state where the member strength is greater than the working load. $G<0$ indicates an unsafe state where the member strength is less than the working load. As mentioned in (1), if the safety index is defined by Equation 1.2.3, and if $R$ and $S$ are independent on each other, and if follow the normal distribution, the probability of failure can be obtained by Equation 1.2.4. 
In addition, if the mean values of $R$ and $S$ are $\mu_{R}$ and $\mu_{S}$ and the standard deviations are $\sigma_{R}$ and $\sigma_{S}$ respectively, then

$$
\begin{aligned}
& \mu_{\mathrm{G}}=\mu_{\mathrm{R}}-\mu_{\mathrm{S}} \\
& \sigma_{\mathrm{G}}=\sqrt{{\sigma_{R}}^{2}+{\sigma_{S}}^{2}}
\end{aligned}
$$

thus, Equation 1.2.3 becomes

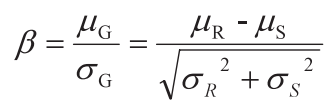

as shown in Figure 1.2.2. By introducing the target safety index $\beta_{0}\left(\beta \geq \beta_{0}\right)$ for a member, and based on Equation 1.2.8,

$$
\mu_{\mathrm{R}}-\mu_{\mathrm{S}} \geq \beta_{0} \sqrt{\sigma_{R}^{2}+\sigma_{S}^{2}}
$$

can be written. Subsequently, the separation factors $\alpha_{R}$ and $\alpha_{S}$ (variation ratios of the input variables with respect to the variation of $G$ ) are used to dispose of the square root, and Equation 1.2.9 can be decomposed into a term associated with the member strength and a term associated with the load

$$
\mu_{\mathrm{R}}-\alpha_{\mathrm{R}} \beta_{0} \sigma_{R} \geq \mu_{\mathrm{S}}+\alpha_{\mathrm{S}} \beta_{0} \sigma_{\mathrm{S}}
$$

The separation factors always take positive values and can be defined by the following equations

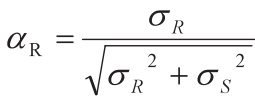

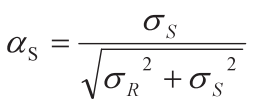

Next, if the design points of the member strength and load are $\left(R^{*}, S^{*}\right)$ when the target safety index becomes $\beta_{0}$, then $R^{*} \geq S^{*}$, therefore, from Equation 1.2.10,

$$
\begin{aligned}
& R^{*}=\mu_{\mathrm{R}}-\alpha_{\mathrm{R}} \beta_{0} \sigma_{R} \\
& S^{*}=\mu_{\mathrm{S}}+\alpha_{\mathrm{S}} \beta_{0} \sigma_{S} \\
& \frac{R^{*}-\mu_{\mathrm{R}}}{\sigma_{R}}=-\alpha_{\mathrm{R}} \beta_{0} \\
& \frac{S^{*}-\mu_{\mathrm{S}}}{\sigma_{S}}=\alpha_{\mathrm{S}} \beta_{0}
\end{aligned}
$$

Assuming that the design points $\left(R^{*}, S^{*}\right)$ were multiplied by a factor of nominal value $R_{n}$ and $S_{n}$, respectively, $\left(R^{*}, S^{*}\right)$ can be expressed as follows

$$
\begin{aligned}
& R^{*}=\phi R_{n} \\
& S^{*}=\gamma S_{n}
\end{aligned}
$$


Here, $\phi$ and $\gamma$ are a resistance factor and a load factor, respectively, which express the partial safety factors of the member strength and the load, respectively. By substituting Equations 1.2.17 and 1.2.18 into Equations 1.2.13 and 1.2.14, the following equations are obtained

$$
\begin{aligned}
& \mu_{\mathrm{R}}-\alpha_{\mathrm{R}} \beta_{0} \sigma_{R}=\phi R_{n} \\
& \mu_{\mathrm{S}}+\alpha_{\mathrm{S}} \beta_{0} \sigma_{S}=\gamma S_{n}
\end{aligned}
$$

Furthermore, by using the target safety index $\beta_{0}$ and each nominal value of $R_{n}$ and $S_{n}$, the resistance factor $\phi$ and the load factor $\gamma$ are derived as follows

$$
\begin{aligned}
& \phi=\left(1-\alpha_{\mathrm{R}} \beta_{0} V_{R}\right) \frac{\mu_{\mathrm{R}}}{R_{n}} \\
& \gamma=\left(1+\alpha_{\mathrm{S}} \beta_{0} V_{S}\right) \frac{\mu_{\mathrm{S}}}{S_{n}}
\end{aligned}
$$

Here, $V_{R}$ and $V_{S}$ are variation coefficients (standard deviation / mean value) of $R$ and $S$ respectively.

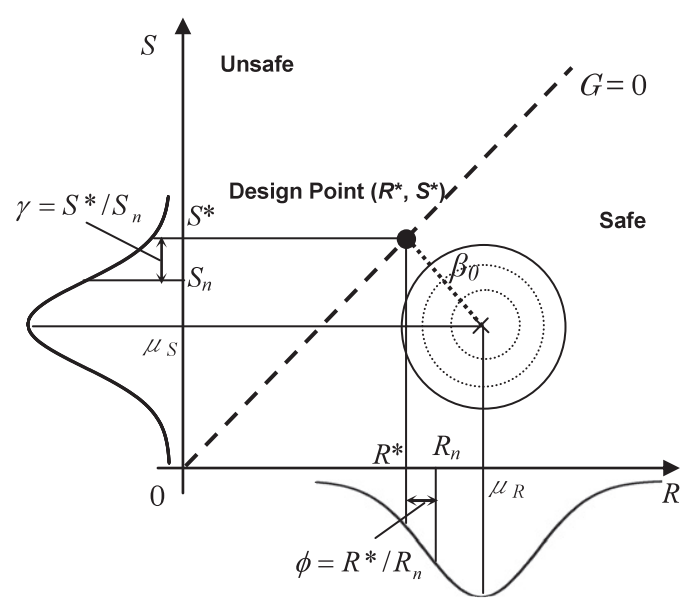

Figure 1.2.2 Relation between the design point of the $R-S$ coordinates and the partial safety factor[8]

This design methodology, which is based on the resistance factor and the load factor, which are derived from the above-mentioned calculation. The multiplication of the nominal values of member strength and the working load by the safety factor, is called the Load and Resistance Factor Design Method (Limit State Design Method), which is commonly used as a practical method. In the field of building structures, this is used for the limit state design method and an index [8-10] regarding load and external forces.

\subsubsection{Calculation Method of Safety Index}

The second moment method is used for calculating the safety index. In the second moment method, $G\left(x_{1}, x_{2}, \cdots, x_{n}\right)$ is a limit state function which is defined with respect to the input parameters $\left\{x_{1}, x_{2}, \cdots, x_{n}\right\}$. If the mean value $\mu_{G}$ and standard deviation $\sigma_{G}$ are 
obtained, then the safety index can be directly obtained from Equation 1.2.3. The following describes the Advanced First Order-Second Moment Method (AFOSM).

In AFOSM, all input parameters are converted into normal distributions, all of which are independent of each other, and the shortest distance from the origin of the converted coordinates to the limit state plane $(G=0)$ is defined as the safety index. Therefore, among the combinations of the input parameters for which the limit state function is $G=0$, it is necessary to find the combination with largest occurrence probability (Most Probable Failure Point, MPFP), but MPFP is normally unknown at the beginning of calculation. In this regard, Rackwitz[14] has proposed an algorithm for searching this point in conjunction with the calculation of safety index.

1) Definition of Safety Index and Most Probable Failure Point (MPFP)

Here, $G\left(x_{1}, x_{2}, \cdots, x_{n}\right)$ is a limit state function defined with respect to $n$ - parameters $\left\{x_{1}, x_{2}, \cdots, x_{n}\right\} . G>0$ indicates a safe state and $G<0$ indicates an unsafe state. The failure point is a combination of input parameters, which is written as

$$
G\left(x_{1}, x_{2}, \cdots, x_{3}\right)=0
$$

The number of combinations of input parameters that constitute a limit state is infinite. Among them, the combination that is most likely to occur is given when the joint probability density function

$$
p d f\left(x_{1}, x_{2}, \cdots, x_{n}\right)=p d f\left(x_{1}\right) p d f\left(x_{2}\right) \cdots p d f\left(x_{n}\right)
$$

of the input parameters is the largest. Then, the combination of $\left\{x_{1}, x_{2}, \cdots, x_{n}\right\}$ is called Most Probable Failure Point $\left\{x_{1}^{*}, x_{2}^{*}, \cdots, x_{n}^{*}\right\}$. The safety index is defined as a normalized distance whose deviation from the mean value $\left\{\mu_{x_{1}}, \mu_{x_{2}}, \cdots, \mu_{x_{n}}\right\}$ of the parameter is divided by the standard deviation $\left\{\sigma_{x_{1}}, \sigma_{x_{2}}, \cdots, \sigma_{x_{n}}\right\}$ of the parameter.

$$
\beta=\sqrt{\sum_{i}\left(\frac{x_{i}^{*}-\mu_{x_{i}}}{\sigma_{x_{i}}}\right)^{2}}
$$

Figure 1.2.3 illustrates a simple example with two input parameters. The two axes of the figure represent the parameters $x_{1}$ and $x_{2}$ as variables which have been standardized by dividing their deviation from mean value by the standard deviation. The origin of the coordinate represents the mean value. The distance from the origin to MPFP is the safety index defined in Equation 1.2.25. 


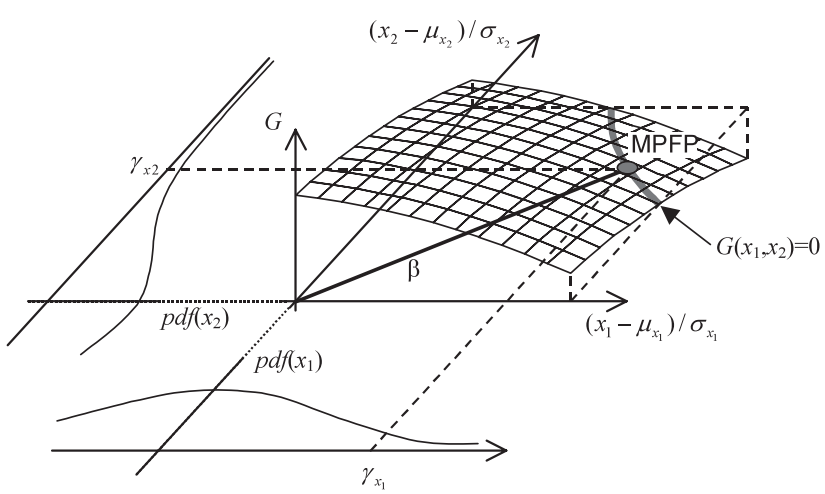

Figure 1.2.3 Definition of the limit state function and MPFP

2) the Rackwitz Algorithm

As the limit state function is nonlinear with its parameters, an iterative calculation method is used to obtain MPFPoint. First, by assuming an appropriate initial guess of $\operatorname{MPFP}\left\{\hat{x}_{1}, \hat{x}_{2}, \cdots, \hat{x}_{n}\right\}$, the limit state function is approximated by Taylor expansion around the initial value. As shown in Figure 1.2.3, when there are two variables, it is equivalent to use the tangent plane of $G$ at the initial guess

$$
G\left(x_{1}, x_{2}, \cdots, x_{n}\right)=G\left(\hat{x}_{1}, \hat{x}_{2}, \cdots, \hat{x}_{n}\right)+\sum_{i} \frac{\partial G}{\partial x_{i}}\left(x_{i}-\hat{x}_{i}\right)
$$

The variance of $G$ can be expressed as follows

$$
\sigma_{G}^{2}=\sum_{i}\left(\frac{\partial G}{\partial x_{i}}\right)^{2} \sigma_{x_{i}}^{2}
$$

Therefore, the safety index becomes

$$
\beta=\frac{\mu_{G}}{\sigma_{G}}=\frac{G\left(\mu_{\mathrm{x}}\right)}{\sqrt{\sum_{i}\left(\frac{\partial G}{\partial x_{i}}\right)^{2} \sigma_{x_{i}}^{2}}}
$$

Equation 1.2.28 indicates that the sum of the variation influences equivalent to the portion of the standard deviation of each variable indicates the changes in the safety index. The separation factor defined by the following equation is the ratio representing how much safety index is affected by the unit variation of variable $x_{i}$. In Figure 1.2.4, this is equivalent to determining the direction of $\beta$ as the direction perpendicular to the intersection line of the tangent plane of $G$ and a plane containing the $x_{1}$ and $x_{2}$ axes.

$$
\alpha_{i}=\frac{\frac{\partial G}{\partial x_{i}} \sigma_{x_{i}}}{\sqrt{\left(\frac{\partial G}{\partial x_{i}}\right)^{2} \sigma_{x_{i}}^{2}}}
$$

By replacing each variable with $\beta$, the following equation can be written

$$
x_{i}^{*}=\mu_{i}+\beta \alpha_{i} \sigma_{x_{i}}
$$


By substituting this expression into Equation 1.2.26, limit state function is expressed only by $\beta$. Therefore, $\beta$ can be obtained from the condition $G(\beta)=0$. For example, as shown in Figure 1.2.4, $\beta$ can be defined using Newton's Method to search for $G(\beta)=0$.

After $\beta$ is obtained, MPFP can be calculated using the following equation

$$
x_{i}=\mu_{i}+\beta \alpha_{i} \sigma_{i}
$$

By updating initial guess of MPFP by calculated MPFP, the whole process is repeated until calculated MPFP converge.

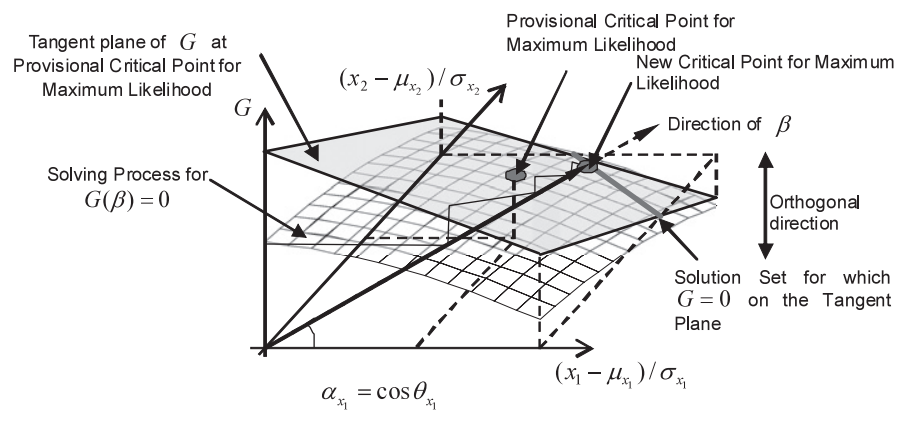

Figure 1.2.4 Calculation procedures for the Rackwitz algorithm

3) Conversion of non-normal distributions

In AFOSM, the input parameters are limited to normal distribution. It is necessary to convert non-normal distributions to equivalent normal distribution. The equivalent normal distribution is determined so that the probability density and probability of excess is identical between original and equivalent normal distribution.

In this research, some of the distribution will be given by lognormal distribution $(g=\ln (x)$ follows normal distribution). The probability density function of $g(=\ln (x))$ is expressed by

$$
g(y)=\frac{1}{\sqrt{2 \pi} \sigma_{L e}} \exp \left\{-\frac{\left(y-\mu_{L e}\right)^{2}}{2 \sigma_{L e}{ }^{2}}\right\} \quad(-\infty<y<\infty)
$$

where $\mu_{L e}$ and $\sigma_{L e}$ are mean and standard deviation of g. From the relation $f(x) d x=g(y) d y$, the probability density function of $x, f(x)$ is

$$
f(x)=\frac{1}{\sqrt{2 \pi} \sigma_{L e} x} \exp \left\{-\frac{\left(\ln x-\mu_{L e}\right)^{2}}{2 \sigma_{L e}{ }^{2}}\right\}
$$

From this equation, the mean value $\mu$ of $x$ is

$$
\mu=\int_{0}^{\infty} x f(x) d x=\exp \left(\mu_{L e}+\frac{1}{2} \sigma_{L e}^{2}\right)
$$

Likewise, the standard deviation $\sigma$ of $x$ is

$$
\sigma=\int_{0}^{\infty}(x-\mu)^{2} f(x) d x=\left\{\exp \left(2 \mu_{L e}+\sigma_{L e}^{2}\right)\right\}\left\{\exp \left(\sigma_{L e}^{2}\right)-1\right\}
$$


The equivalent normal distribution refers to a normal distribution in which both the probability density and the excess probability coincide at $x^{*}$, as shown in Figure 1.2.5.

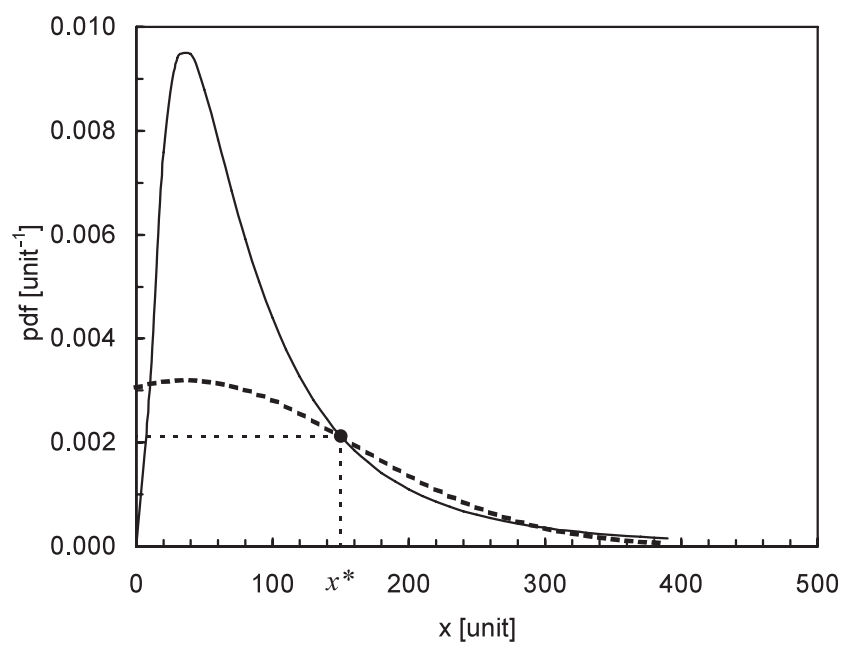

Figure 1.2.5 Conversion of logarithmic normal distribution to equivalent normal distribution

By using the mean value $\mu$ and the standard deviation $\sigma$ of $x$ on the original logarithmic normal distribution, the mean value $\mu_{e q}$ and the standard deviation $\sigma_{e q}$ on the equivalent normal distribution at the design point $x *$ can be given as:

$$
\begin{aligned}
& \mu_{e q}=x_{i}\left\{1-\ln \left(x_{i}\right)+\mu\right\} \\
& \sigma_{e q}=x_{i} \times \sigma
\end{aligned}
$$

\subsubsection{Review of Previous Works using Theory of Structural Reliability Engineering}

Some of the AIJ (Architectural Institute of Japan)'s academic Standards[8-11] make use of the limit state design format. The limit state design method aims at achieving target reliability for ultimate and serviceability limit states of a structure. The partial safety factors are determined as load factor and strength reduction factor. In this design format, determination of target reliability is one of the key issues. Kanda et al.[15] proposed to set target safety index based on the principle of minimizing total expected life-cycle cost. Kohno[16] has proposed optimum design reliability level calculated based on expected total cost minimization which takes into consideration of the cost associated with the earthquake damage level. Also, Kohno et al.[17] proposed the method of setting optimum design reliability level based on in order to achieving the target performance which takes into account economic factor related to order of building owners. Then the target performance is defined as the maximum story drift angel yielded from an earthquake load. Furthermore, Kohno[18] proposed target design reliability levels of various use of buildings based on the proposed method of total cost minimization. Hirata et al.[19] used the strength of structure as an index based on construction cost in order 
to represent the structural reliability for seismic actions.

In the field of fire safety engineering, Magnusson et al.[20] performed reliability analysis with respect to evacuation safety of commercial complex by using AFOSM and Monte Carlo simulations. The results were presented by the safety index and corresponding partial safety factors of the input variables. By using the technique, Frantzich et al.[21] established a comparison framework for the evacuation safety performance of multiple design proposals. Mizono et al.[22][23][24] studied the variation of the input variables and the accuracy of the prediction formulas used in the verification method of evacuation safety in Building Standards Law of Japan. After obtaining the safety index implied in the verification method, they proposed a combination of appropriate partial safety. Regarding structural fire resistance, Magnusson et al.[25] performed reliability analysis of fire resistive steel structures and proposed a practical design format. Suzuki et al.[26] performed reliability analysis using Monte Carlo simulations with respect to the fire resistance performance of the steel columns and beams in a fully-developed fire, and analyzed variation of the safety index with respect to collapse in two scenarios with and without considering the fire occurrence ratio.

As mentioned above, in the field of building structures, systematic methods such as the limit state design method have been established at least in theoretical basis. Research on practical development is undertaken to properly set the target safety index. On the other hand, a proper design system has not been established yet in the field of fire resistance in Japanese engineering society.

\subsection{Objective and Composition of this Study}

This study focuses on the problems associated with the verification method described in Section 1.1.3. The safety level with respect to localized fire and a fully-developed fire in a typical building space is analyzed by using the theory of reliability engineering. The purpose of this is to quantitatively express the safety level implied in the Verification Method and to clarify the sensitivity of each input parameter on the safety level archived. Also, it aims at establishing an appropriate method for setting a safety factor for each parameter by quantitatively presenting the safety index which is implicitly contained as a nominal value of the Verification Method.

To do this, this thesis comprises 8 chapters as described in Figure 1.3.1. Chapters 2 and 3 are to prepare for main analysis analyzing the technical contents of Performance Verification Method for Fire Resistance. Chapters 4 and 5 refer to the analysis of the safety level with respect to localized fire. Chapters 6 and 7 refer to the analysis of the safety level with respect to a fully-developed fire. Finally, Chapter 8 summarizes the conclusion of the findings obtained from this study. 


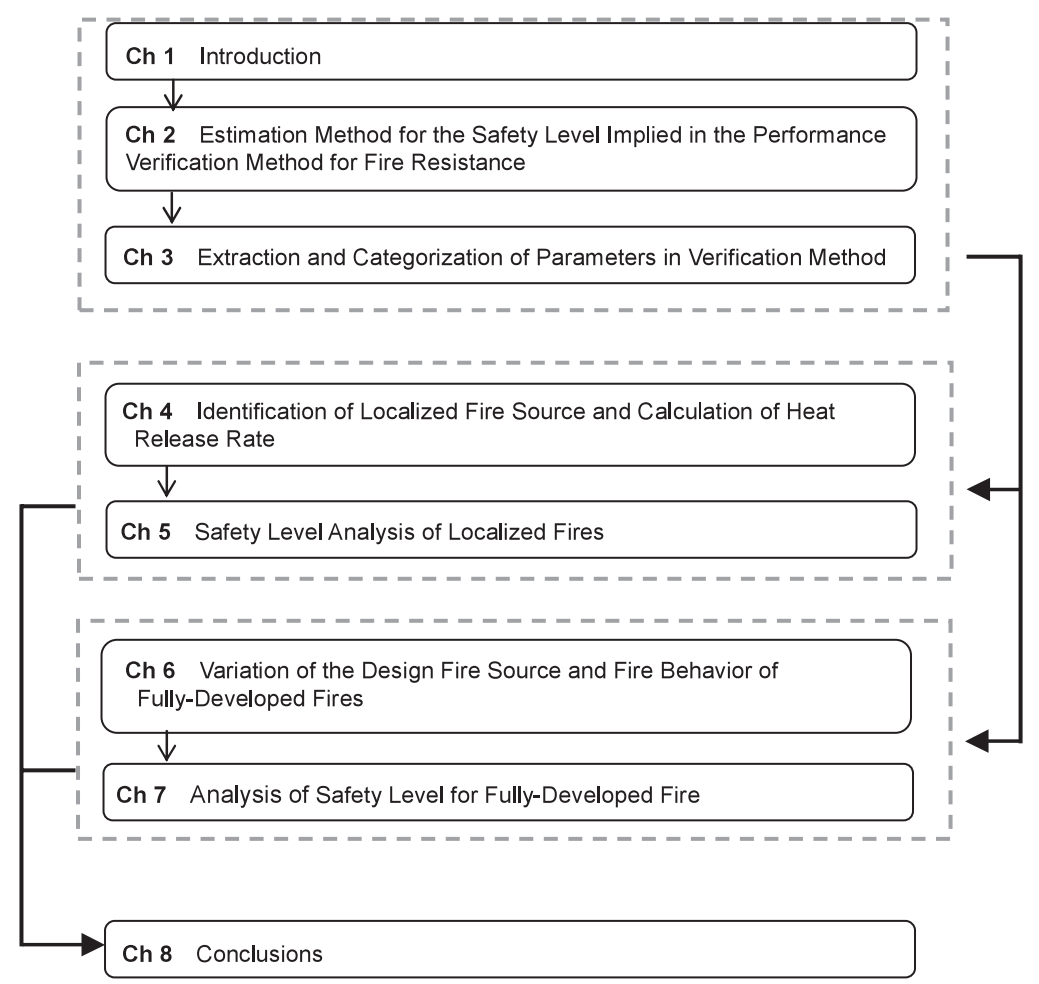

Figure 1.3.1 Composition of this thesis

Chapter 1 refers to the background and the purpose of this study and its relation with previous studies. The theory of structural reliability engineering used in the field of building structures is described as a method to represent the safety level quantitatively. As to the analytical method used throughout this paper, Advanced First-Order Second-Moment (AFOSM) method is briefly described.

Chapter 2 refers to the estimation method of the safety level with respect to localized and fully-developed fires as stipulated in the Verification Method. First of all, the framework of the Verification Methods are described. Then the calculation formulas for steel elements under localized and fully-developed fires are described. Secondly, in order to analyze the safety level, a couple of definitions are made specific to steel elements on such as the limit state, limit state function and an unsafe state.

Chapter 3 refers to the extraction of the parameters associated with the calculation formula of the critical temperature and the maximum temperature of the steel members. Firstly, the parameters are categorized into two groups according to the statistical variations intrinsic to each parameter and the prediction errors (uncertainties) of the calculation formula. Secondly, with respect to the categorized input parameters, the statistical and experimental data are collected to describe the variability and uncertainty by using probability density functions of either normal or lognormal distribution. 
Chapter 4 refers to the parameters associated with the behavior and duration of localized fire. To do this, firstly published experimental data related to various combustible objects are corrected. A methodology is proposed to predict the heat release rate (HRR) curve of various combustibles. HRR could be evaluated by names or by physical information (e.g. constituent material and dimensions). As an example, a hotel lounge layout was assumed. A possible fire source for localized fire was estimated from a hotel brochure, and a set of sofas is used as the fire source of maximum burning. Using the estimation method based on the name of the material, the heat release rate curve is estimated for the set of sofas. After the heat release rate curve is calculated by adjusting the time to spread to adjacent items, the variation of the fire duration is expressed by a probability density function of normal distribution.

Taking the hotel lounge as an example, Chapter 5 refers to the estimation and the analysis of the safety level with respect to localized fire by using a non-insulated steel column located close to a fire source in the case of localized fire. The variations and uncertainties in the input parameters (obtained from Chapters 3 and 4) are considered in the formulas in order to estimate the safety index by AFOSM method. In order to identify the safety level achievable by satisfying the Verification Method requirements, the dimensions of a steel member which suitably satisfies said requirements is specified. The safety index and sensitivity are analyzed for the specified elements. By summarizing the MPFP, the partial safety factor of each of the input parameters are clarified and compared with the nominal values implied in the Verification Method. For generalization, the variation of the safety index is analyzed by changing the specification of column element. The safety index and the partial safety factors are correlated. This information would be useful to fulfill target safety index by way of partial safety factors.

Chapter 6 refers to parameters associated with fully-developed fires. The stochastic variations and the uncertainties are quantified in fire load density, the correction factors for the fire duration and fire temperature rise and the correction factor for the formula of equivalent fire duration. Regarding the fire load density, the existing survey results are collected and statistically processed to develop the probability density function of lognormal distribution. As for the correction factors, correction factors were derived from the comparison with full-scale fire experiments of compartmental fire. The results are expressed by lognormal distribution. The estimation error of the equivalent fire duration is represented by normal distribution.

Chapter 7 refers to the analysis of the safety level with respect to fully-developed fire. As an example, insulated steel columns and beams in a typical office was considered. The variations and uncertainties of the input parameters are expressed by the results in Chapters 3 and 6. The safety index is analyzed with AFOSM method. Similar to Chapter 5 for localized fires, the safety index was estimated for the combinations of parameters that satisfy the Verification Method requirements. The size of openings, the cross-sectional dimensions of steel members are identified. Then the safety index and corresponding partial safety factors were analyzed. As a second step, the results were 
generalized to derive the relationship between target safety index and partial safety factors on major input parameters.

Chapter 8 summarizes the findings from this research.

\section{REFERENCES}

1. The Building Center of Japan, Total Fire Safety System of Buildings, 1988 (in Japanese)

2. Ministry of Land, Infrastructure and Transportation, Verification method for fire resistance, Notification 1433, 2000 (in Japanese)

3. K. Harada, Performance Based Codes and Fire Safety Design of Buildings, Journal of Japan Association for Fire Science and Engineering, Vol. 48(1), pp.1-6, 1998 (in Japanese)

4. K. Harada et al., Performance Evaluation Methods for Evacuation Safety and for Structural Fire Resistance, Third International Conference on Performance Based Codes and Fire Safety Design Methods, pp. 212-223, 2000

5. Ministry of Land, Infrastructure and Transport and Tourism, Guidance and Examples in Performance Verification Method for Fire Resistance, Inoue Shoin, 2001 (in Japanese)

6. M. Ichikawa, Structural Reliability Engineering - Reliability Method for Strength Design and Life Prediction, Kaibundo, 1996 (in Japanese)

7. Japanese Standards Association, Glossary of Terms Used in Dependability, JIS Z8115, 1981 (in Japanese)

8. Architectural Institute of Japan, Standard for Limit State Design of Steel Structures (draft), 1990 (in Japanese)

9. Architectural Institute of Japan, Recommendation for Limit State Design of Steel Structures, 1998 (in Japanese)

10. Architectural Institute of Japan, Recommendations for Loads on Buildings, 1993 (in Japanese)

11. Architectural Institute of Japan, Recommendations for Limit State Design of Buildings, 2002 (in Japanese)

12. A. Nishitani and T. Kashimura, Reliability Index and Second Moment Method (Technical Information: Design using the Basis of Confirmed Reliability and Reliability Index, part 3), Journal of Architecture and Building Science 114 (1441), 1999 (in Japanese)

13. T. Takada and T. Takahashi, Design Methods for Load and Resistance Factors (Technical Information: Design using the Basis of Confirmed Reliability and Reliability Index, Part 4), Journal of Architecture and Building Science, No. 114 (1441), 1999 (in Japanese)

14. A. H-S. Ang and W. H. Tang., Probability Concepts in Engineering Planning and Design Volume II - Decision, Risk, and Reliability, John Wiley \& Sons, Inc., 1984 
15. J. Kanda and T. Hirakawa, Optimum Target Reliability Index for Design Loads in Limit State Design, Journal of structural and construction engineering. Transactions of AIJ, No. 523, pp.39-46, 1999 (in Japanese)

16. M. Kohno, Design Reliability Level considering Earthquake Damage Costs, Summaries of Technical Papers of Annual Meeting Architectural Institute of Japan, 1996 (in Japanese)

17. M. Hattori, M. Igarashi and M. Kohno, Determination of Target Performance Level of Steel Buildings by Cost Minimization, Summaries of Technical Papers of Annual Meeting Architectural Institute of Japan, 1997 (in Japanese)

18. M. Kohno, Determination of Target Performance Level of Buildings by Cost Minimization, Summaries of Technical Papers of Annual Meeting Architectural Institute of Japan, 1998 (in Japanese)

19. K. Hirata and T. Ishikawa, Probabilistic Evaluation of Target EarthquakeResistant Level Derived from Requirements of Users: For better indication of performance reflecting user's needs, Journal of structural and construction engineering. Transactions of AIJ, No. 543, pp. 23-29, 2001 (in Japanese)

20. S. E. Magnusson, H. Frantzich and K. Harada, Fire Safety Design Based on Calculations : Uncertainty Analysis and Safety Verification, Department of Fire Safety Engineering Lund Institute of Technology Lund University, Report 3078, 1995

21. M. Angerd and H. Frantzich, The Use of Uncertainty Analysis in Performance Based Design, Proceedings of the 4th International Conference on PerformanceBased Codes and Fire Safety Design Methods, pp. 179-189, 2002

22. M. Mizuno et al., A Consideration on the Safety Factors Implemented in Characteristic Input Values and Prediction Formula Determined in Verification Method for Egress Safety From a Fire Floor: An analysis of target safety level for egress during building fires Part 1, Journal of Architecture and Planning. Transactions of AIJ, No. 563, pp. 1-8, 2003 (in Japanese)

23. M. Mizuno et al., Target Safety Level of Egress from Fire Room in Typical Office Buildings: an analysis of target safety level for egress during building fires Part 2, Journal of environmental engineering. Transactions of AIJ, No. 566, pp.17-24, 2003 (in Japanese)

24. M. Mizuno et al., Target Safety Level of Egress from Fire Floor in Typical Office Buildings: an analysis of target safety level for egress during building fires Part 3, Journal of environmental engineering. Transactions of AIJ, No. 573, pp. 25-32, 2003 (in Japanese)

25. S. E. Magnusson and O. Pettersson, Rational Design Methodology for Fire Exposed Load Bearing Structures, Fire Safety Journal, Vol. 3, pp. 227-241, 1980/81

26. J. Suzuki et al., Reliability Analysis of the Steel Structural Member in a Fire, Journal of structural and construction engineering. Transactions of AIJ, No. 48B, pp. 111-118, 2002 (in Japanese) 